\title{
CREADOR Y CRIATURA: PERCEPCIÓN DE PROFESORES DE ESPAÑOL SOBRE LA PRODUCCIÓN DE MATERIAL DIDÁCTICO
}

\author{
CREATOR AND CREATURE: SPANISH TEACHERS' \\ PERCEPTIONS ABOUT THE PRODUCTION OF TEACHING \\ MATERIAL
}

\author{
Ana Karla Pereira de MIRANDA ${ }^{1}$ \\ Álvaro José dos Santos GOMES ${ }^{2}$ \\ Daniela Sayuri Kawamoto KANASHIRO ${ }^{3}$
}

\begin{abstract}
Resumen: Nuestro estudio forma parte del proyecto de investigación acerca de la formación de profesores de español del Grupo de Estudos e Pesquisas em Linguagem e Educação (Geple), de la Universidade Federal de Mato Grosso do Sul (UFMS). En este trabajo, discutimos la percepción de tres alumnas del curso de Letras acerca de sus experiencias como productoras de materiales didácticos para cursos modulares con fines académicos, vinculados al Programa Idiomas sem Fronteiras (IsF). Fundamentamos nuestros análisis en Leffa (2007), Vetromille-Castro (2011), Paiva (2011) y Morin (2016), cuando discutimos la educación lingüística, los Sistemas Adaptativos Complejos (SAC) y la producción de materiales didácticos. Los aspectos metodológicos llevados a cabo en la selección y análisis de los datos están basados en la investigación cualitativa, mediante aplicación de cuestionario semiestructurado (DUARTE, 2004; GIL, 2010). Los análisis se organizan en seis bloques: (i) acerca de las informantes; (ii) análisis del contexto; (iii) desarrollo del material; (iv) implementación del material; (v) evaluación del material; (vi) formación docente. Por fin, consideramos que la experiencia de elaboración de material didáctico, bajo la orientación del coordinador pedagógico, fue significativa para la formación de las profesoras, pues les posibilitó nuevos aprendizajes y la vivencia del protagonismo docente.
\end{abstract}

Palabras clave: Formación docente. Idiomas sem Fronteiras. Español como Lengua Extranjera. Sistemas Adaptativos Complejos.

\begin{abstract}
Our study is part of the project about Spanish teacher education related to the Grupo de Estudos e Pesquisas em Linguagem e Educação (Geple), from Universidade Federal de Mato Grosso do Sul (UFMS). In this paper, we discuss the perception of three Portuguese and Spanish Education students about their experiences as teaching material productors for modular courses with academic purposes, bound to Programa Idiomas sem Fronteiras (IsF). Our analysis is founded in Leffa (2007), Vetromille-Castro (2011), Paiva (2011), Morin (2016), regarding linguistic education, complex adaptive system and the elaboration of teaching materials. The methodological procedures employed for data selection and analysis are based on the principles of qualitative research, by applying a semi-structured questionnaire. The analysis is organized in six blocks: (i) about the informants; (ii) context analysis; (iii) material development; (iv) material implementation; (v) material evaluation; (vi) teacher education. At last, we consider that the experience of elaborating teaching materials, under the guidance of the pedagogical coordinator, was relevant for the education of the teachers, as it fostered new learnings and the practice of teacher protagonism.
\end{abstract}

Keywords: Teacher education. Language without borders. Spanish as a Foreign Language. Complex adaptive system.

\footnotetext{
1 Universidade Federal de Mato Grosso do Sul (UFMS), Campo Grande, Mato Grosso do Sul, Brasil; ana.miranda@ufms.br; https://orcid.org/0000-0002-2151-0021

2 Universidade Federal de Mato Grosso do Sul (UFMS), Campo Grande, Mato Grosso do Sul, Brasil; alvaro.brt@gmail.com; https://orcid.org/0000-0002-9123-7729

3 Universidade Federal de Mato Grosso do Sul (UFMS), Campo Grande, Mato Grosso do Sul, Brasil; daniela.ead.ufms@gmail.com; https://orcid.org/0000-0001-5017-1420
} 
- Creador y criatura: percepción de profesores de español sobre la producción de material didáctico

\section{Introducción}

El presente estudio objetiva discutir la percepción de tres profesoras de lengua española, aún en formación, acerca de sus experiencias en la elaboración de materiales didácticos con fines específicos. Durante el grado, los estudiantes del curso de Letras de la Universidade Federal de Mato Grosso do Sul (UFMS), campus de Campo Grande, tenían, entre las varias oportunidades académicas, la de actuar como profesores en los cursos presenciales de lenguas del Programa Idiomas sem Fronteiras (IsF), bajo la orientación de un docente de la institución. Para eso, se seleccionaban alumnos que se presentaban a convocatorias divulgadas durante el año, conforme las plazas disponibles.

El programa brasileño IsF, del Ministerio de Educación (MEC), se constituyó por medio de la ordenanza $n^{0}$ 1.466, de 18 de diciembre de 2012 (BRASIL, 2012), inicialmente con el nombre de Inglês sem Fronteiras. Tenía, entre otros objetivos, el de desarrollar acciones para la internacionalización de la enseñanza superior a través de la consolidación de una política lingüística de las instituciones acreditadas y de la formación de profesores de lenguas extranjeras.

La internacionalización "[...] en nivel nacional, sectorial e institucional se define como el proceso en lo cual se integra una dimensión internacional, intercultural o global en los propósitos, funciones y oferta de educación en nivel superior"4 (KNIGHT, 2003, p. $2^{5}$ apud COURA; COURA, 2017, p. 3). Es decir, la internacionalización es una integración de enseñanza, investigación y servicios, cuya necesidad es resultante de una sociedad globalizada (AMORIM; FINARDI, 2017).

Conforme mencionamos, el IsF debería contribuir para ese proceso de internacionalización. El programa estaba organizado por Núcleos de Lenguas (NL) en diferentes universidades públicas. Cada NL debía planificary desarrollar la oferta de cursos modulares con fines académicos. Los alumnos de los cursos de formación de profesores de idiomas elaboraban materiales didácticos e impartían clases, según mencionamos, bajo la orientación de un coordinador pedagógico que debía ser un docente de la institución. También se ofertaban cursos en línea de inglés, alemán e italiano, además de los cursos presenciales de esos idiomas y de otros como español, francés, japonés y de portugués para extranjeros. Los NL eran igualmente responsables de la aplicación de

4 Todas las traducciones son versiones libres nuestras. En el original: "[...] em nível nacional, setorial e institucional é definida como o processo no qual se integra uma dimensão internacional, intercultural ou global nos propósitos, funções e oferta de educação pós secundária".

$5 \mathrm{KNIGHT}$, J. Updating the definition of internationalization. Internacional Higher Education, Fall, n. 33, 2003. 
pruebas de competencia y examen de nivel. Es importante evidenciar que, en 2020, el Programa IsF se desvinculó del MEC y, desde entonces, está relacionado con la Associação dos Dirigentes das Instituições Federais de Ensino Superior (Andifes), identificado ahora como Red Andifes IsF. Cabe igualmente mencionar que a causa de la necesidad del aislamiento físico determinado por la pandemia de Covid-19, en 2020, la forma de oferta de cursos sufrió cambios.

En la UFMS, las actividades del IsF empezaron en 2013 con la aplicación de exámenes de dominio, el Test of English as a Foreign Language (TOEFL). En 2014, iniciaron los cursos presenciales de inglés y, a finales de 2017, el de español y el de portugués para extranjeros. También se desarrollaron cursos de francés de 2016 a 2018, cuando la institución tenía un lector de la Embajada de Francia. Desde 2020, la UFMS no forma parte de la Red Andifes IsF.

Nuestro foco, en esta investigación, es la formación docente en lo que se refiere a la experiencia en producción de material didáctico con fines académicos. Para eso, fundamentamos el estudio en las contribuciones teóricas de Vetromille-Castro (2011), Paiva (2011), Morin (2016) y Souza (2019) respecto a la formación de profesores, el lenguaje como un sistema complejo y métodos de enseñanza bajo estas perspectivas; y de Eres Fernández (2000) y Leffa (2007) acerca de la producción de materiales didácticos para la enseñanza de lengua extranjera. A continuación, analizamos la percepción de tres estudiantes del curso que impartieron clases en el Programa IsF. Concluimos el estudio en las consideraciones finales, cuando evidenciamos que la experiencia fue significativa en lo que se refiere a la formación de las estudiantes en cuanto profesoras de español, sobre todo debido a la oportunidad que tuvieron de vivenciar el protagonismo docente en su propia práctica.

\section{Marco teórico}

La formación académica se constituye por un conjunto de procedimientos que involucra varias etapas. Como un sistema complejo, compuesto de muchos elementos que se relacionan y se ajustan, la formación académica no siempre es resultado del todo formativo, las partes no representan el todo y el todo no puede ser representado por la suma de las partes (MORIN, 2016). En ese contexto, disyuntivo y complejo, el reto que se plantea es de ¿cómo la formación inicial docente es posible y viable considerando la multiplicidad de elementos que componen dicho sistema? O aún, ¿cómo planificar un curso de formación inicial docente frente a la complejidad de elementos que se interrelacionan? El eje de esas cuestiones se relaciona con la forma que el alumnado y el 
- Creador y criatura: percepción de profesores de español sobre la producción de material didáctico

profesorado aprenden y enseñan a la vez (FREIRE, 1996) ${ }^{6}$, así como su percepción sobre lengua y cultura (SOUZA, 2019). En este apartado, proponemos una discusión reflexiva acerca de esas inquietudes que se nos presentan a diario en los múltiples contextos de la formación inicial docente: el desafío de aprender y de enseñar lenguas.

Es corriente en los cursos de formación docente de las universidades, en sus componentes curriculares, asignaturas que buscan, por medio de métodos y enfoques específicos, proponer una reflexión acerca del proceso de aprendizaje del alumno, o sea, de qué modo él aprende y cómo lo hace. Una u otra vez, en los cursos de grado, los profesores formadores intentan llevar a cabo en sus clases una aproximación entre el método de enseñanza y el aula, relacionándolo al estudiante, profesor y al contenido, como si fuera posible reproducir artificialmente un sistema tan heterogéneo como es el espacio escolar, que es dinámico, abierto y extremadamente complejo en lo que se refiere a las relaciones interpersonales. Dicha cercanía, es una de las muchas estrategias para que los futuros profesores tengan experiencias en el campo práctico de la docencia asimilando las teorías de aprendizaje antes de las pasantías, asignaturas obligatorias en los cursos de formación de profesores. Queda claro, sin embargo, a lo largo de las investigaciones científicas en el área de la Pedagogía, que no hay única fórmula o un manual de instrucciones para lograr éxito en el proceso de aprendizaje (GATTI, 2010).

Los procesos de enseñanza y aprendizaje durante un largo período, y basados en teorías educativas, concibieron el aula como un espacio privilegiado para la concretización de conocimientos. El espacio interactivo, destinado a tal efecto, se presenta en el imaginario de profesores, alumnos y comunidad escolar, como un lugar caótico y, en gran medida, complejo. La indisciplina, la vida palpitante de los alumnos, la maraña de acciones intermitentes, la dificultad de asimilar los contenidos, entre una miríada de otros factores, corroboran para la visión de que el aula sea un caos o algo complejo (VETROMILLE-CASTRO, 2011). Esa visión del aula, aunque los términos utilizados no dialoguen directamente con conceptos científicos, no es del todo errónea, sin embargo, está bastante alejada de los principios del caos y de la complejidad.

En el campo de la Lingüística Aplicada (LA), bajo el diálogo y la perspectiva de los Sistemas Adaptativos Complejos (SAC), sabemos que la adquisición de una segunda lengua, por ejemplo, no ocurre de una misma forma o siguiendo un patrón didáctico o metodológico, lo que conlleva a pensar el modo por lo cual concebimos la propia lengua

6 Para Freire (1996), el profesor aprende al enseñar y el alumno enseña al aprender. Así que se comprende que enseñar no es una transferencia de contenidos como si solo el docente conociera todo y otros nada supieran. 
y el proceso de aprendizaje (LARSEN-FREEMAN, 1997). Durante muchos años y por mucho tiempo, hemos visto que la enseñanza de lenguas tradicionalmente está basada en distintos métodos y enfoques, sea el audiolingual, directo, traducción, entre tantos otros; la reproducción de los métodos implica pensar que todos los alumnos aprenden de la misma forma o al mismo tiempo. En los presupuestos teóricos de los SAC, lo cual representa un giro epistemológico en el campo científico (MORIN, 2016), el aula, el propio lenguaje y todos los fenómenos de aprendizaje se relacionan al principio del caos y de la complejidad ${ }^{7}$. Para Vetromille-Castro (2011, p 118, nuestra traducción $\left.{ }^{8}\right)$,

Los estudiantes utilizan una variedad de recursos, como diccionarios, lectura de libros, música y películas y, en un entorno de aprendizaje virtual, consultan los foros, comentan los portafolios, leen textos en línea, con frecuencia y secuencias aleatorias. No hay forma de predecir que, siguiendo los pasos $\mathrm{X}, \mathrm{Y}, \mathrm{Z}$, el alumno aprenderá. El aprendizaje, en general, no sigue un orden, una secuencia de pasos preestablecidos y que se encaja en todo y cualquier sistema educativo.

En la misma línea, según Paiva (2011), se puede predecir mínimamente que, en contextos formales de aprendizaje, ya sea en la modalidad presencial ya sea a distancia, los estudiantes serán capaces de memorizar reglas y vocabulario, sin embargo, no podemos predecir si todos adquirirán el idioma, es decir, si ellos van a empeñarse en prácticas sociales de lenguaje mediadas por la lengua extranjera. Si bien no podemos predecir el conocimiento de la lengua meta que un alumno adquirirá durante su vida, podemos predecir que, en promedio, será más competente si tiene la oportunidad de usar la lengua en contextos auténticos. Así que la adquisición no es predecible y varía según la naturaleza de las interacciones entre todos los elementos del sistema de adquisición del aprendiz.

7 La teoría del caos es un campo de estudio en matemáticas, con aplicaciones en varias disciplinas, incluyendo la Física, la Ingeniería, la Economía, la Biología y la Filosofía. La teoría del caos se ocupa de sistemas complejos y dinámicos estrictamente deterministas, pero que presentan un fenómeno fundamental de inestabilidad Ilamado sensibilidad a las condiciones iniciales que, al modular una propiedad de recurrencia adicional, los hace impredecibles en la práctica a largo plazo. (MORIN, 2015)

8 En el original: "Os alunos lançam mão de recursos variados, como dicionários, leitura de livros, músicas e filmes, e, em ambiente virtual de aprendizagem, consultam aos fóruns, comentários em portfólios, leitura de textos on-line, com frequência e sequências aleatórias. Não há como prever que, seguindo os passos $X, Y, Z$, o aluno aprenderá. A aprendizagem, de um modo geral, não acompanha uma ordem, uma sequência de passos pré-estabelecidos e que se encaixa em todo e qualquer sistema educacional.". 
- Creador y criatura: percepción de profesores de español sobre la producción de material didáctico

Aunque la palabra caos, en su uso actual, también se refiere a una expresión de la vida cotidiana, que está directamente relacionada con la desorganización, la imprevisibilidad y la inestabilidad, en el ámbito científico, el caos es un requisito previo para el orden (OLIVEIRA, 2011). La teoría del caos aquí utilizada dialoga con los conceptos formulados en los campos de la Física, Matemáticas y de la Filosofía, en los que las condiciones iniciales de un sistema dan como resultado infinitas posibilidades y combinaciones. Los supuestos teóricos del caos y SAC se aplican al contexto del aprendizaje y del aula fundamentalmente porque las condiciones iniciales tienen un fuerte impacto en las acciones posteriores. La autoorganización, a través de la emergencia de nuevos comportamientos como resultado de las múltiples interacciones, es lo que mejor define este proceso. Para Morin (2016, p. 59, nuestra traducción ${ }^{9}$ ), "[...] la complejidad comprende efectivamente el tejido de eventos, acciones, interacciones, retroacciones, determinaciones, acasos que conforman nuestro mundo fenomenal". El caos establece el desequilibrio inicial, el sistema se autoorganiza promoviendo un equilibrio, luego y a partir de la influencia del entorno (sistema abierto), se puede desequilibrar nuevamente, generando otros comportamientos, para que a continuación se reequilibre (MORIN, 2015). Lo que define el equilibrio de este sistema es la condición inicial y la interacción de las relaciones entre los entornos internos y externos. En resumen, el aula es caótica no porque sea un choque de fuerzas opuestas que provoquen un desequilibrio ad aeternum, sino porque presenta patrones de comportamiento predecibles desde las condiciones iniciales y porque tiene autoorganización a partir de esa configuración.

Por una parte, es innegable el hecho de que hemos avanzado en la comprensión de los fenómenos de adquisición de segunda lengua y lengua adicional. Teorías de la LA y de otras áreas nos permitieron, con más claridad, comprender el lenguaje en su concepción dinámica y viva: el contexto práctico de su uso efectivo, de modo único, por el hablante. Por otra parte, y desafortunadamente, si evolucionamos en la comprensión de dicho fenómeno, no hemos sido capaces de articular el conocimiento acerca de la lengua con el universo del aprendizaje. ¿Cómo pensar un método para algo que no es replicable? ¿Cómo personalizar varios métodos de acuerdo con las características del alumnado considerando el aspecto bancarizado de la educación ${ }^{10}$ ? ¿Cómo preparar al

9 En el original: "[...] a complexidade compreende, efetivamente, o tecido de acontecimentos, ações, interações, retroações, determinações, acasos que constituem o nosso mundo fenomenal."

10 La educación bancaria es una concepción del educador brasileño Paulo Freire, y criticada por él, que designa al proceso de enseñanza basado en una concepción de que los alumnos son "vasijas" o recipientes que deben ser "Ilenados" por el educador. En la obra Pedagogía del Oprimido (FREIRE, 2019), en el segundo capítulo titulado "La concepción bancaria de la educación como instrumento de opresión", el autor analiza la forma poco democrática como las instituciones de enseñanza basan sus métodos partiendo del supuesto de que el alumnado no posee conocimientos y que el acto educativo es demasiado pasivo, centrado en una narrativa única, en este caso la del profesor. 
nuevo profesor de lenguas bajo esa perspectiva? Todas las preguntas se relacionan con el papel del profesor, ¿̇mediador, curador, gerente de interacción?

Con respecto a esta nueva concepción de lenguaje, método y aprendizaje de lenguas extranjeras, poniendo de relieve también las voces de otros autores que comparten esta comprensión del fenómeno lingüístico, consideramos que las reflexiones de Souza (2019, p. 249, nuestra traducción"1) acerca de los objetivos de enseñanza son, a la vez, más relevantes y necesarios que los propios métodos. El autor sugiere que "[...] lo que más importa no es el método $X, Y$ o Z, sino para quién estamos enseñando, para qué y cuáles son los recursos disponibles". El conocimiento de la audiencia se relaciona con la perspectiva de lo que quieren aprender y de lo que pueden ofrecer de conocimiento previo para contribuir con la enseñanza y con su propio aprendizaje. Lo que hemos visto, en el marco de la crítica de las teorías pedagógicas, es que hay un alejamiento entre el propio concepto de lenguaje, la realidad formativa disociada de ese concepto y la ausencia de conocimiento de las características del alumnado. El triple aspecto cuando está desarticulado promueve la construcción de barreras que se vuelven difíciles de ser superadas, sobre todo en el campo de la formación inicial docente.

Este es otro problema: la cuestión no es el método, la cuestión es el concepto de lengua. El método, que es el "cómo hacerlo", es irrelevante, porque una vez que tenemos el concepto de lo que es y lo que no es la lengua, podemos utilizar técnicas de varios métodos para lograr nuestro objetivo. [...] el concepto de lengua ha cambiado. Ahora estamos hablando de translingualismo, cómo usar la lengua de forma fluida, etc. Eso es un cambio en el concepto de lengua. ${ }^{12}$ (SOUZA, 2019, p. 248-249).

En ese sentido, conforme Souza (2019), en los cursos de formación, se debería preparar profesores protagonistas, que fueran capaces de analizar quiénes son sus alumnos, cuál es su contexto de actuación, cuáles son las necesidades de ese entorno y de los estudiantes, cuáles son los recursos disponibles para, a partir de ahí, poder definir lo que se puede hacer y desarrollar en la enseñanza. Para él, eso es más importante que cualquier método.

11 En el original: "[...] o que mais importa não é o método $X, Y$ ou $Z$, mas sim para quem estamos ensinando, para que e quais são os recursos disponíveis.".

12 En el original: "Esse é outro problema: a questão não é o método, a questão é o conceito de língua. O método, que é o "como fazer", é irrelevante, porque uma vez que temos o conceito do que é e do que não é a língua, podemos lançar mão de técnicas de vários métodos para atingir o nosso objetivo. [...] o conceito de língua mudou. Agora estamos falando de translingualismo, como se usar a língua de forma fluida, etc. Isso é mudança no conceito de língua.". 
- Creador y criatura: percepción de profesores de español sobre la producción de material didáctico

A partir de eso, preguntamos: ¿¿Dónde empieza la formación inicial docente? Este interrogante es un camino posible para que podamos reflexionar acerca de las experiencias formativas en los cursos de grado. ¿El proceso que se lleva a cabo para formar nuevos profesores se inicia tan solo en los bancos de las universidades? ¿Cuáles experiencias anteriores del aprendiz podría formar parte de este futuro docente? ¿Hay habilidades que se deben considerar? Todas estas preguntas entretejen un complejo entramado de prácticas en el tejido de la formación inicial docente. Con las perspectivas teóricas de los SAC, basado aún en los campos de la LA respecto a la lengua, el universo formativo se expande y forma parte de la complejidad del sistema. El reto que se nos afronta es justamente, sabiendo de estas características, ¿ cómo preparar al nuevo docente para los desafíos de enseñar lengua?

Para Sabota (2011), aprender a ser profesor es un proceso que conlleva la complejidad en su esencia. Aunque todos los profesores que quieran enseñar idiomas en contextos formales pasen por la misma etapa, o sea, la formación inicial en un curso de Letras, la diversidad de conocimientos previos que esos estudiantes traen desencadenará una serie de implicaciones, a partir de las cuales, habrá una gran variedad en su formación individual. La autora destaca que la actuación del profesor se compone no solamente de los aspectos profesionales, sino también de todo lo que le constituye como un ser humano. De ahí, sus valores, creencias, identidad, sentimientos, entre otros aspectos, se involucran en su construcción como profesor.

Considerando la formación docente como un sistema complejo, Sabota (2011) propone ocho categorías que forman parte de ese fractal, a saber: contexto socio histórico; aspectos políticos; input teórico; creencias; expectativas; aspectos propios de la interacción; experiencia previa con el alumnado de lengua extranjera; factores bio-cognitivos y afectivos. De ese modo, observamos que sujetos que participan del mismo proceso formativo pueden tener percepciones distintas sobre ese momento, dependiendo no solamente de sus experiencias y vivencias, sino también de cómo las reciben.

En los estudios de Gatti (2010) y Gatti e Barreto (2009), que investigan las políticas docentes en Brasil, se encontró que en el análisis de los planes de estudios de las asignaturas de formación profesional (metodologías y prácticas de enseñanza, por ejemplo), también predominan tan solo referenciales teóricos sin asociación con prácticas educativas y, en la gran mayoría de los cursos analizados, se abordan de forma genérica o superficial. En el mismo sentido, identificaron que una "[...] parte de estas titulaciones promueve especialización temprana en aspectos que podrían abordarse en especializaciones o posgrados, o que, claramente, apuntan a la formación de un profesional distinto al 
profesor"13 (GATTI, 2010, p. 1374). A esta realidad se añade a lo que hemos discutido, la formación inicial docente es un proceso lo cual necesita ser revisado desde los objetivos de lo que sea enseñar y aprender bajo la complejidad de este fenómeno.

Aunque este apartado teórico trajo más interrogantes que caminos posibles y efectivos, consideramos que ellas, las preguntas, son relevantes porque forman parte de las preocupaciones que se nos han surgido, como, por ejemplo, cómo preparar mejor a nuestros alumnos, o de generar discusiones acerca de cómo instruirlos a preparar de modo más adecuado materiales didácticos o qué enfoque adoptar para ello. En esta investigación, basados en los marcos teóricos y en los resultados que presentamos, creemos que pensar el aula y el aprendizaje como un SAC nos permite ver al fenómeno educacional sin moldes, barreras o métodos fijos, y que la formación inicial docente debe estar relacionada con las múltiples posibilidades de un organismo vivo y pulsante que es la sala de clase.

Otro aspecto importante del proceso educativo, que también es parte integrante de los SAC, son los materiales didácticos. Según el documento oficial brasileño, las Orientações Curriculares Nacionais para o Ensino Médio - OCEM (BRASIL, 2006, p. 154, nuestra traducción ${ }^{14}$ ), material didáctico “[...] es un conjunto de recursos de los cuales el profesor se vale en su práctica pedagógica", entre ellos, un libro didáctico, textos, vídeos, gramáticas, diccionarios.

La elaboración de materiales didácticos es un importante tema que debe ser contemplado en los cursos de formación docente. Aunque el profesor de la educación básica trabaje con un libro de texto, normalmente distribuido por el Programa Nacional do Livro Didático (PNLD), o con cuadernillos, bastante comunes en escuelas particulares brasileñas, no raras veces, tendrá que elaborar alguna actividad para complementar determinado aspecto previsto en el curso.

De acuerdo con las OCEM (BRASIL, 2006), es importante considerar el libro de texto como una de las posibilidades de los recursos pedagógicos, no el único. Además de eso, Silva (2005, p. 182) afirma que no hay teorías y tampoco métodos de aprendizaje de lenguas "que expliquen o que den la fórmula definitiva de cómo aprender una lengua, con una estrategia infalible" y Salaberri Ramiro (1990, p. 109) añade que "ningún libro se

13 En el original: "[...] parte dessas licenciaturas promove especialização precoce em aspectos que poderiam ser abordados em especializações ou pós-graduação, ou que, claramente, visam a formação de outro profissional que não o professor."

14 En el original: "[...] é um conjunto de recursos dos quais o professor se vale na sua prática pedagógica". 
- Creador y criatura: percepción de profesores de español sobre la producción de material didáctico

adapta totalmente a una situación de aprendizaje concreta", es decir, aunque el profesor utilice un libro de texto en sus clases, no hay uno que atienda a todas las necesidades de los alumnos. De ese modo, consideramos concerniente que los profesores sean capaces de construir o de adaptar un material didáctico que sea lo más adecuado posible a los propósitos del curso y a los objetivos de aprendizaje de los alumnos.

Es importante aclarar que la producción en el área de español tuvo un boom en los años 90, conforme explica Eres Fernández (2000), en consecuencia de la creciente demanda de los cursos, resultado de la valoración de ese idioma en el mercado laboral. En Brasil, hubo aumento en la publicación de libros de texto, de profesiones y oficios, de ejercicios, de lectura, de apoyo gramatical, diccionarios, materiales lúdicos, para el autoaprendizaje, de cultura y literatura, además de revistas y publicaciones periódicas (ERES FERNÁNDEZ, 2000).

Sin embargo, cuando se trata de fines específicos, la necesidad de crear o adaptar todas las actividades didácticas es aún más frecuente, ya que no tenemos tantas opciones de libros de texto, en el ámbito editorial, que versen sobre fines académicos. En ese caso, tenemos que incluir también otros materiales didácticos como vídeos, informes, artículos, carteles, resúmenes para eventos académicos, etc. Bedin (2017) afirma que un libro de texto con fines generales no atiende satisfactoriamente a cursos con fines específicos (académicos y profesionales) una vez que esos tienen propósitos bastante distintos que exigen el desarrollo de temas y conocimientos lingüísticos acorde a ese ámbito.

Leffa (2007) afirma que la elaboración de materiales didácticos debe suponer por lo menos cuatro etapas que forman un ciclo: análisis, desarrollo, implementación y evaluación. En la primera etapa, hay que considerar las necesidades de los estudiantes. Según Leffa (2007, p. 16, nuestra traducción ${ }^{15}$ ), "Lo que el alumno ya sabe debe servir de andamio para que él alcance lo que aún no sabe. Nadie aprende algo que es totalmente conocido, ni algo que sea totalmente nuevo.". En el segundo momento - el desarrollo - se definen: los objetivos, conforme las necesidades de los alumnos, el enfoque, el contenido, las actividades, los recursos y la secuencia de las actividades. En la tercera etapa, la de implementación, vamos a considerar que el material será usado por el propio profesor, ya que también podría ser utilizado por otro docente o por el alumno sin la presencia de un profesor como ocurre en los cursos autoinstructivos. En el momento de la implementación, el autor del material/profesor normalmente añade comentarios orales, durante la clase, acerca de las tareas, de modo que la observación de algún problema en

15 En el original: "O que aluno já sabe deve servir de andaime para que ele alcance o que ainda não sabe. Ninguém aprende algo que é totalmente conhecido e nem algo que seja totalmente novo." 
la comprensión o en el desarrollo de las propuestas permite que estas sean reformuladas posteriormente. La evaluación, último momento, puede realizarse informalmente o por medio de cuestionarios, entrevistas o protocolos. Los resultados de la evaluación deben retroalimentar el proceso, es decir, subsidiar el desarrollo de la reelaboración del material.

\section{Metodología}

En esta investigación se buscó analizar la percepción de alumnos del curso de grado en Letras Portugués y Español en cuanto a la producción de materiales didácticos para fines académicos. Para tanto, se seleccionaron los sujetos de la investigación, representados por tres alumnas que impartieron clases en el programa IsF y que produjeron sus propios materiales didácticos. El período de esta experiencia fue de octubre de 2017 hasta julio de 2019. En el cuadro siguiente, en que se describe la organización de los cursos de español, las nombramos como informantes 1,2 y 3.

Cuadro 1. Cursos de español desarrollados en el programa IsF/UFMS ${ }^{16}$

\begin{tabular}{|c|c|c|c|}
\hline Informantes & Curso & $\begin{array}{l}\text { Carga } \\
\text { horaria }\end{array}$ & Periodo \\
\hline Informante 1 & $\begin{array}{l}\text { Competências Interculturais em Contexto } \\
\text { Acadêmico de Língua Espanhola }\end{array}$ & 16 & $\begin{array}{l}\text { Oferta } 2 \text { - } 2017 \\
23 / 10 \text { a } 21 / 11\end{array}$ \\
\hline Informante 1 & $\begin{array}{l}\text { Competências Interculturais em Contexto } \\
\text { Acadêmico de Língua Espanhola }\end{array}$ & 16 & $\begin{array}{l}\text { Oferta } 3-2017 \\
27 / 11 \text { a } 21 / 12\end{array}$ \\
\hline Informante 2 & $\begin{array}{l}\text { Competências Interculturais em Contexto } \\
\text { Acadêmico de Língua Espanhola }\end{array}$ & 16 & $\begin{array}{l}\text { Oferta } 1-2018 \\
26 / 3 \text { a } 18 / 4\end{array}$ \\
\hline Informante 2 & Bem-vindo ao Espanhol: Língua Internacional & 32 & $\begin{array}{l}\text { Oferta } 2-2018 \\
30 / 4 \text { a } 26 / 6\end{array}$ \\
\hline Informante 2 & Espanhol para Eventos Internacionais & 32 & $\begin{array}{l}\text { Oferta } 5-2018 \\
13 / 8 \text { a } 13 / 10\end{array}$ \\
\hline Informante 2 & Bem-vindo ao Espanhol: Língua Internacional & 32 & $\begin{array}{l}\text { Oferta } 7 \text { - } 2018 \\
22 / 10 \text { a } 18 / 12\end{array}$ \\
\hline Informante 3 & Espanhol para Acolhimento & 32 & $\begin{array}{l}\text { Oferta } 3-2019 \\
30 / 4 \text { a } 25 / 6\end{array}$ \\
\hline Informante 3 & $\begin{array}{l}\text { Compreensão leitora de textos acadêmicos em } \\
\text { espanhol }\end{array}$ & 16 & $\begin{array}{l}\text { Oferta } 5 \text { - } 2019 \\
1 / 7 \text { a } 4 / 7\end{array}$ \\
\hline
\end{tabular}

Fuente: Elaboración propia de los autores.

16 El cuadro presenta los datos de los cursos ofertados hasta julio de 2019, porque realizamos la aplicación del cuestionario acerca de la percepción de las informantes en ese período. Sin embargo, conviene aclarar que la informante 3 desarrolló otros cursos hasta diciembre del referido año. 
- Creador y criatura: percepción de profesores de español sobre la producción de material didáctico

Los datos fueron recolectados mediante la aplicación de un cuestionario semiestructurado en la plataforma Google Forms. La técnica elegida para la recogida de datos, a partir del cuestionario semiestructurado, permite, al mismo tiempo, la libertad de expresión del entrevistado y el mantenimiento del enfoque por parte del investigador (GIL, 2010). Duarte (2004, p. 215, nuestra traducción ${ }^{17}$ ) añade un razonamiento similar, para la autora:

La entrevista o cuestionario semiestructurado es un recurso metodológico que busca, con base en teorías y supuestos definidos por el investigador, recolectar respuestas a partir de la experiencia subjetiva de una fuente, seleccionada por tener informaciones que se desea conocer.

El cuestionario constaba de 18 preguntas divididas en siete apartados, que son: 1) presentación - descripción del objeto investigado e invitación a participar de la encuesta; 2) información personal - reporte de experiencias previas en la producción de materiales didácticos; 3) análisis - conocimiento previo de las características de los alumnos que iban a utilizar el material didáctico producido; 4) desarrollo - identificación de la percepción del proceso de elaboración del material didáctico; 5) implementación - feedback sobre la calidad del material producido y su aplicación; 6) evaluación - identificación de la percepción del estudiante sobre la calidad del material producido; 7) formación docente - percepción de las entrevistadas sobre el marco general de la formación inicial docente como productoras de materiales didácticos.

Aunque los datos obtenidos en esta perspectiva metodológica no nos permita compilarlos en una estadística, como suele ser en las investigaciones de naturaleza cuantitativa, consideramos que incluso el componente metodológico de esta investigación dialoga con la orientación teórica del trabajo en cuanto a la formación inicial docente: un sistema adaptativo complejo, abierto, dinámico, que sufre interferencias externas, caótico, que se retroalimenta, que posibilita nuevas emergencias y que se autoorganiza. Estas características están presentes y señaladas en la discusión de los resultados presentados en el próximo apartado. En síntesis, cada participante posee una visión distinta de la formación inicial docente y de la producción de materiales didácticos.

17 En el original: "A entrevista ou questionário semiestruturado é um recurso metodológico que busca, com bases em teorias e pressupostos definidos pelo investigador, recolher respostas a partir da experiência subjetiva de uma fonte, selecionada por deter informações que se deseja conhecer". 


\section{Análisis de datos}

Según mencionamos, el formulario presentaba preguntas con posibilidades de selección de respuestas y otras con espacios para escribir los comentarios. Estaba organizado en las cuatro etapas de preparación de materiales, propuestas por Leffa (2007), además de una primera parte acerca de datos personales y la última, sobre formación docente.

\section{Sobre las informantes}

En cuanto a los datos de ese apartado, estos se refieren a las experiencias previas de las participantes de la investigación con relación a la docencia antes de ingresar en el IsF. Como mencionado, analizamos las respuestas de tres alumnas del curso de Letras Portugués y Español que actuaron como profesoras de cursos ofrecidos en dicho Programa.

Entre las tres, dos ya habían tenido experiencias en cursos libres, una en escuelas de idiomas particulares y en el Programa de Extensão de Ensino de Línguas (Progeli) de la UFMS y otra solamente en este último. La informante 2, por su vez, tuvo experiencias con la docencia en el ámbito del curso de Letras durante las asignaturas prácticas.

En cursos libres, normalmente se usan materiales propios de cada empresa. En el Progeli, se suele utilizar libros de texto de editoriales extranjeras, no específicos a estudiantes brasileños. De ese modo, ninguna de las informantes había tenido experiencia en elaboración de materiales didácticos, sobre todo en contexto tan específico, como cursos con fines académicos.

\section{Elaboración de materiales. Etapa 1 - Análisis}

Es importante resaltar que cuando empiezan a elaborar el curso, las becarias no conocen el público. Los cursillistas, muchas veces, poseen un dominio variado de la lengua, aunque los cursos tengan un nivel específico. Eso ocurre porque hay estudiantes que recurren al IsF para repasar contenidos o mantener su contacto con la lengua meta. Además, la exigencia para que se inscriban es que tengan la competencia mínima para acompañar las clases, pero no hay impedimentos si tienen un dominio superior a lo exigido.

Para Leffa (2007), el examen de las necesidades de los estudiantes, de sus deseos, expectativas y preferencias es parte importante de la elaboración del material didáctico y debe ser realizado previamente. En el caso de nuestra experiencia, las profesoras no 
- Creador y criatura: percepción de profesores de español sobre la producción de material didáctico

tenían contacto con los cursillistas antes de que empezara la oferta, imposibilitando este conocimiento anterior del público. Sin embargo, todas mencionaron que el hecho de no conocer a sus alumnos antes de preparar el material no fue un aspecto dificultador. Posiblemente, esa constatación esté relacionada con que los cursos tenían objetivos muy específicos acerca de estudios en ámbitos académicos, tales como, comprensión lectora de textos académicos, español como lengua internacional, eventos académicos, etc. En ese sentido, el programa de los cursos fue un recurso fundamental para guiarlas en la etapa de elaboración, como se explica más adelante.

A ese respecto, la informante 3 señala que, aunque no sea un aspecto dificultador, no conocer a los alumnos puede obstaculizar el estudio del profesor para la preparación del material. Para ella, ese estudio debe ser más abarcador cuando no se conoce al público. De ese modo, observamos que la profesora traza estrategias para remediar esa carencia, o sea, de cierta manera, demuestra su protagonismo en cuanto docente, como señala Souza (2019).

También destacamos que, debido al hecho de no conocer al público en un momento anterior a la oferta, en los materiales, se formulaban algunas actividades con un carácter nivelador, o sea, tareas que permitían a las profesoras conocer la etapa del aprendizaje de sus estudiantes y adaptar la propuesta a su nivel. Así, aunque pensemos que conocer al público podría haber facilitado el trabajo de las docentes en la tarea de preparar un material que fuera específico a sus alumnos, sus respuestas indican que trazaron otras estrategias para suplir esa ausencia, tales como apoyarse en el programa de los cursos y hacer una planificación más abarcadora, lo que revela que, en ese aspecto, las profesoras fueron protagonistas de su práctica.

\section{Elaboración de materiales. Etapa 2 - Desarrollo}

Conforme resaltamos, el programa del curso resultó ser fundamental como directriz para la elaboración de los materiales didácticos. Dos participantes mencionaron que: "Conocer el objetivo de la oferta hace el trabajo de investigación para la producción de material más puntual"18. Otra informante argumentó que ese documento "[...] garantiza directrices de cómo elaborar el material didáctico"19. Además de eso, el hecho de ofrecer un curso tan específico también determina un público, formado por alumnos, docentes y técnicos, con objetivos igualmente específicos.

18 En el original: "Conhecer o objetivo da oferta torna mais pontual a pesquisa para a produção do material." 19 En el original: "[...] garante diretrizes de como elaborar o material didático". 
Al respecto del programa, este abarca los más diferentes aspectos del curso, de modo a estandarizar las ofertas en los distintos lugares donde ocurren en Brasil. Entre los elementos que figuran en el programa están: objetivos; justificación; metodología; contenidos (funcionales, lingüísticos, culturales, etc.); recursos; evaluación y referencias. Dado su carácter orientador, observamos que ese recurso actuó como un facilitador en lo que se refiere al desarrollo del material didáctico.

Conforme Leffa (2007), el desarrollo del material didáctico presupone la definición de: i) los objetivos; ii) el enfoque; iii) el contenido; iv) las actividades; v) los recursos; vi) el orden de las actividades y vii) la cuestión de la motivación. En el referido programa, los objetivos, el contenido y el enfoque, aunque este último de manera indirecta, ya estaban establecidos por el Núcleo Gestor (NG). Cabía a las profesoras, de esa manera, seleccionar/elaborar las actividades y los recursos, así como ordenarlos de modo a facilitar el aprendizaje y a motivar a los estudiantes.

En cuanto a eso, todas afirmaron que utilizaron libros de texto, gramáticas, recursos didácticos de la internet, materiales auténticos (vídeos, textos, etc.) y diccionarios. Además, una participante utilizó materiales elaborados por otros profesores. Señalamos que estos últimos se refieren a los materiales elaborados por otras becarias en ofertas anteriores. Esta preocupación de las autoras demuestra un cuidado especial con los contextos auténticos de aprendizaje de lenguas y con la diversidad de fuentes que pueda contribuir para ampliar el abanico de posibilidades de enseñanza, lo que dialoga con lo que propone Paiva (2011) acerca de las estrategias de enseñanza y aprendizaje.

Todas consideraron que la orientación del coordinador pedagógico contribuyó para la elaboración de los materiales. En ese sentido, destacamos el papel de la supervisión en la formación del profesor, que sirve para facilitar el acceso a conocimientos sobre el proceso educativo, así como ayuda en la creación de objetivos y estrategias para que dicho proceso suceda. Para Zabalza (2014), las prácticas hechas sin asistencia pierden, en parte, su carácter formativo y tienen reducida su capacidad de originar nuevos significados y de garantizar competencias positivas. De ese modo, para que las experiencias prácticas con la docencia contribuyan, de hecho, para la formación del profesor, la supervisión tiene un papel importante, pues por medio de ella: se ajustan los propósitos formativos; se potencia la sinergia entre los involucrados; se controlan las posibles desviaciones del plan central, entre otros.

Entre las dificultades encontradas, relacionaron: sintetizar el material considerando el grupo bastante heterogéneo en lo que se refiere al conocimiento del idioma y al origen del curso (había alumnos de diferentes áreas); saber la cantidad de actividades suficientes 
- Creador y criatura: percepción de profesores de español sobre la producción de material didáctico

para cada clase; adecuar el material a la propuesta del curso, que era el español en contexto académico. Aunque las becarias ya hubieran tenido alguna experiencia previa como docentes, es importante mencionar que se trataba de otros contextos. O eran cursos libres o cursos de idiomas del Progeli, donde, por lo general, se utilizan libros de texto. En el caso de los grupos del IsF, las profesoras en formación tenían que construir todo el material con poquísimas referencias, ya que no es común encontrar libros de texto que traten de cómo elaborar resúmenes, hacer presentaciones orales en eventos científicos, escribir artículos académicos. Además de eso, volvemos a mencionar que la dificultad de adecuación del material a la propuesta del curso puede estar relacionada al hecho de que los alumnos eran siempre de carreras diferentes con distintos conocimientos del idioma. Así que el contenido del material debería contemplar también amplias áreas de formación.

\section{Elaboración de materiales. Etapa 3 - Implementación}

En el caso de la experiencia en el IsF, la implementación del material se hace por el propio autor que lo elaboró, o sea, el profesor en formación. Es, por ese motivo, un recurso que se explica oralmente a los alumnos y que funciona en distribución complementaria con el profesor, conforme alude Leffa (2007). En cuanto a su adecuación, dos personas consideraron que el material didáctico estaba muy adecuado a los objetivos del curso y una informante juzgó que estaba un poco adecuado. En el caso de que el material no esté adecuado, Leffa (2007) señala que los errores más grandes o los malentendidos que dificultan la implementación pueden ser apuntados o reformulados para una nueva edición. Sin embargo, en el caso de nuestra experiencia, pocas veces se repitió el curso.

Al respecto de ajustes a lo largo de las clases, una participante tuvo que modificar o adaptar el material durante el desarrollo del curso y explicó que tuvo que añadir más actividades. Otra manifestó que propuso actividades de refuerzo, según la necesidad del grupo. Eso, en su percepción, no era una adaptación o modificación del material producido. En cuanto al aspecto relativo a la implementación, es importante percibir el aula como un SAC, pues a partir del comportamiento de los elementos (alumnos, profesores, materiales didácticos, entre otros factores), emergen nuevos atractores en el sistema, promoviendo nuevos comportamientos, lo que resta claro que las principales características de este fenómeno es la autoorganización y la retroalimentación del sistema. De ahí, que las autoras tuvieron que adaptar el material o añadir otros elementos para que el funcionamiento del sistema se mantuviese. 
Es posible relacionar el tipo de adaptación hecha por la informante - incremento de actividades - a una de las dificultades encontradas durante el desarrollo de los cursos: saber la cantidad de tareas suficientes para cada clase. En ese sentido, subrayamos la necesidad de una profesionalización docente que no remita solamente a una formación técnica, sino que también sume "[...] la sensibilización y percepción para el contexto, el entorno, las diferencias, las resignificaciones, los cambios" (MONTE MÓR, 2015, p. 39, nuestra traducción $\left.{ }^{20}\right)$. Las profesoras, en nuestra investigación, eran autoras de sus propios materiales didácticos y, de ese modo, necesitaban además de tener un conocimiento técnico, o sea, metodológico, didáctico y lingüístico, saber seleccionar los recursos que serían utilizados, elaborar las actividades - ya que, como hemos dicho, son escasos los materiales para fines específicos -y adaptarse a situaciones no previstas, lo que configura una de las características del SAC.

\section{Elaboración de materiales. Etapa 4 - Evaluación}

Sobre la etapa de evaluación, Leffa (2007) menciona que cuando se trata del trabajo de solo un docente, la evaluación de una propuesta didáctica es, normalmente, informal. El profesor prepara una actividad, aplica, observa, reformula, aplica de nuevo y así sucesivamente. Cuando tenemos a varios docentes que utilizan el mismo material elaborado por compañeros de una institución, se puede hacer una evaluación más formal, por medio de consulta a especialistas, aplicación de cuestionarios o entrevistas con alumnos.

En los cursos desarrollados en el IsF/UFMS, no hubo momentos de evaluación formal del material didáctico, o sea, no aplicamos cuestionarios, tampoco desarrollamos entrevistas, protocolos o informes. Las oportunidades de evaluación se realizaron informalmente, sea por medio de comentarios de la coordinadora pedagógica realizados en los materiales o encuentros de orientaciones y diálogos entre la coordinadora y las alumnas del curso de Letras, participantes del programa, sea por las profesoras en formación observando el uso del material durante las clases, o por medio de comentarios y reacciones del público que utilizó el cuadernillo.

A ese respecto, una informante contestó negativamente sobre la falta de oportunidad para evaluar el material didáctico producido, sea formal o informalmente, por medio de informes, formularios, charlas, etc. Según esa alumna, los materiales siempre

20 En el original: "[...] a sensibilização e percepção para o contexto, o entorno, as diferenças, as ressignificações, as mudanças". 
- Creador y criatura: percepción de profesores de español sobre la producción de material didáctico

pasaban por la aprobación de coordinadores, sin embargo, no se hacía una evaluación acerca de lo que podría mejorar. Esa respuesta nos parece contradictoria, ya que, tras pasar por la aprobación de coordinadores, se sobrentiende una evaluación del material. Además de eso, aspectos relacionados con lo que era posible perfeccionar siempre eran tratados en encuentros de orientaciones.

Los alumnos de los cursos del programa pudieron evaluar el material didáctico producido, según respuesta de dos participantes. Posiblemente, estas profesoras deben haber propiciado discusión acerca de cómo sus estudiantes valoraban las actividades propuestas.

Las informantes tuvieron pocas oportunidades de utilizar más de una vez el material didáctico elaborado, porque impartieron el mismo curso para públicos diferentes. Una de ellas mencionó que su producción sufrió algunos cambios con base en la primera experiencia. Seguramente, si tuviera la oportunidad de utilizarla por tercera vez, algunas actividades pasarían por nuevos ajustes. Conforme Leffa (2007), tenemos un ciclo en el cual la evaluación debe llevar al inicio de una nueva etapa de análisis, desarrollo, implementación y evaluación, de modo sucesivo.

\section{Formación docente}

Preguntamos si juzgaban importante tener una formación específica para elaborar materiales didácticos. Una participante contestó que no y explicó que el curso de grado garantizaba un aporte teórico pertinente para desarrollar los contenidos. Otras respuestas argumentaron que era importante más estudio en el idioma, espacio para testar el material y que la formación específica para aquel caso, sería el grado en Letras. Respuestas distintas para integrantes del mismo curso evocan la idea del aula como un SAC (MORIN, 2015) en la medida que el todo no es la suma de las partes y que las partes no representan el todo, es decir, que el curso de formación inicial docente, aunque tenga el mismo componente curricular, repercutió de modo distinto entre las informantes (SABOTA, 2011) en el momento en que produjeron sus materiales. Evidentemente, si nuestros futuros profesores, no poseen el mismo comportamiento frente al aprendizaje, es de esperar que los alumnos de los cursos del IsF, de un modo general, así también lo tengan.

Entre los elementos propuestos, los que más contribuyeron para el proceso de elaboración de materiales fueron las clases del curso de Letras, la orientación del coordinador pedagógico y el conocimiento de la lengua española. Dos personas señalaron que la experiencia profesional también ayudó en la construcción de materiales didácticos. De ahí, es importante poner de relieve, lo que ya se ha demostrado hace 
mucho, sobre todo en las producciones de Freire $(1996,2019)$, que el papel del profesor pasa por la mediación, curaduría y facilitación del proceso de aprendizaje del alumno que ya viene con sus propias experiencias y percepciones sobre el mundo y sobre lo que es ser profesor.

Todas fueron unánimes en afirmar que la experiencia como elaboradoras de material didáctico contribuyó mucho para su formación profesional.

Informante 1: La producción del material propicia una profundización del conocimiento sobre la lengua, ya sea gramatical o funcional, lo que me convierte en una profesora con más base para ayudar a los alumnos, al mismo tiempo que me hace más segura en mi práctica docente. ${ }^{21}$

Informante 2: Sin duda, ha contribuido mucho. En la escuela donde trabajo actualmente, son los profesores quienes elaboran los materiales didácticos utilizados en las clases, que se actualizan cada dos años. Si quizá me asignan la misma misión, además de ya tener una idea de cómo hacerlo, me sentiré mucho más segura debido a la experiencia adquirida en el IsF.22

Informante 3: Sí, pues la experiencia de elaboración de material didáctico en el IsF fue algo nuevo en mi vida profesional, nunca antes vivida. Fue algo novedoso. Muy provechoso. ${ }^{23}$

Los comentarios evidencian la experiencia significativa de elaboración de material didáctico, en el proceso de formación docente. Todo el desarrollo involucró etapas como analizar el contexto de uso, producir propiamente el material, implementarlo y evaluarlo. En esa trayectoria, hubo interacciones con los alumnos y con la coordinadora pedagógica que también contribuyeron para reflexiones acerca del proceso y de su propia actuación como docente.

21 En el original: “A produção do material propicia um aprofundamento no conhecimento sobre a língua, seja ele gramatical ou funcional, o que me transforma em uma professora com mais embasamento para ajudar os alunos, ao mesmo tempo em que me torna mais segura na minha prática docente."

22 En el original: "Com certeza, contribuiu muito. Na escola onde trabalho atualmente, são os professores quem elaboram os materiais didáticos utilizados em sala de aula, que são reatualizados de dois em dois anos. Se porventura me incumbirem a mesma missão, além de já ter uma noção de como fazer, sentirei muito mais segurança devido à experiência adquirida no IsF."

23 En el original: "Sim, pois a experiência de elaboração de material didático no IsF foi algo novo na minha vida profissional, nunca antes vivida. Foi uma novidade. Muito proveitoso." 
- Creador y criatura: percepción de profesores de español sobre la producción de material didáctico

\section{Consideraciones finales}

En esta sección, sintetizamos algunos aspectos observados en esta investigación. Acerca de las tres profesoras en formación, evidenciamos que ninguna había tenido experiencia en elaborar un material didáctico, implementarlo y evaluarlo.

Sobre las etapas de producción, mencionadas por Leffa (2007) - análisis, desarrollo, implementación y evaluación - observamos que, en la primera, no se caracterizó como un aspecto dificultador, el hecho de que no conocieran el público antes de iniciar el proceso. Eso nos parece contradictorio, sobre todo si consideramos los argumentos del mencionado investigador cuando afirma que es importante conocer el público antes de elaborar el material, hay que considerar las necesidades, expectativas y preferencias de los estudiantes. Sin embargo, las estrategias trazadas por las profesoras para superar esa ausencia demuestran su protagonismo docente.

En cuanto al desarrollo, destacamos que el programa del curso sirvió como directriz que orientó la elaboración del material didáctico y auxilió su planificación. También observamos que el coordinador pedagógico actuó como facilitador del proceso educativo, así como destacamos su papel en lo que se refiere a la supervisión en la formación docente, promoviendo asistencia y reflexiones. Las dificultades encontradas estaban centradas en la especificidad de la propuesta del curso, o sea, el español para fines académicos.

Al respecto de la etapa de implementación, las participantes consideraron que, en general, el material producido estaba adecuado a los objetivos del curso y, algunas veces, hubo la necesidad de hacer ajustes o modificaciones. Señalamos que fueron pocas las oportunidades de repetir un curso, lo que dificultó su reformulación. Además, observamos características del SAC en las prácticas de las profesoras.

Acerca de la etapa de evaluación, el estudio identificó que no hubo momentos específicos con ese objetivo. Sin embargo, informalmente, las profesoras en formación tuvieron la oportunidad de discutir sus propuestas con la coordinadora pedagógica y también con los alumnos. Desafortunadamente, fueron pocas oportunidades de volver a utilizar el mismo material, ya que eso podría propiciar momentos para perfeccionar el trabajo.

Evidenciamos que la percepción de las informantes acerca del proceso de elaboración del material didáctico con fines académicos, desde su uso y adaptación, dialogó con la concepción del aula y la formación inicial docente como un sistema complejo: autoorganizado, no-linear, sensible a las condiciones iniciales, abierto, 
dinámico, que se retroalimenta, que sufre cambios a partir de la emergencia de nuevos comportamientos y que está en constante cambio. Lo que conlleva a la idea de que la formación inicial docente, como propone Sabota (2011), es en parte resultado de un fractal compuesto por varias características y múltiples aspectos.

\section{Referencias}

AMORIM, G. B.; FINARDI, K. R. Internacionalização do ensino superior e línguas estrangeiras: evidências de um estudo de caso nos níveis micro, meso e macro. Avaliação, Campinas, v. 22, n. 3, p. 614-632, nov. 2017. Disponível em: http://www.scielo.br/pdf/aval/ v22n3/1982-5765-aval-22-03-00614.pdf. Fecha de consulta: 14 jun. 2019.

BEDIN, M. C. Espanhol para fins específicos no ensino superior tecnológico e formação docente: articulações, rumos e possibilidades. 2017. Tese (Doutorado em Educação) - Faculdade de Educação, Universidade de São Paulo, São Paulo, 2017. doi:10.11606/T.48.2017.tde-31072017-162103. Fecha de consulta: 13 out. 2020.

BRASIL. Orientações Curriculares para o Ensino Médio. Linguagens, códigos e suas tecnologias. Brasília: Ministério da Educação, Secretaria de Educação Básica, 2006. Recuperado de: http://portal.mec.gov.br/seb/arquivos/pdf/book_volume_01_internet. pdf. Fecha de consulta: 30 jul. 2020.

BRASIL. A internacionalização na Universidade Brasileira: resultados do questionário aplicado pela Capes. Brasília: Ministério da Educação, Coordenação de Aperfeiçoamento de Pessoal de Nível Superior, 2107. Disponível em: https://www.capes. gov.br/images/stories/download/diversos/A-internacionalizacao-nas-IES-brasileiras. pdf. Fecha de consulta: 10 jun. 2019.

BRASIL. Ministério da Educação. Portaria n⿳0 1.466, de 18 de dezembro de 2012. Institui o Programa Inglês sem Fronteiras. Diário Oficial da União: Seção 1, Brasília, DF. 19 dez. 2012. p. 28. Disponível em: http://isf.mec.gov.br/images/pdf/portaria_ normativa_1466_2012.pdf. Fecha de consulta: 30 jul. 2020.

COURA; K. V.; COURA, K. V. Internacionalização do Ensino Superior: razões políticas, econômicas, socioculturais e acadêmicas. In: CONGRESSO INTERNACIONAL ABED DE EDUCAÇÃO A DISTÂNCIA, 23., 2017, Foz do Iguaçu. Anais do $23^{\circ}$ Congresso Internacional ABED de Educação a distância. Disponível em: http://www.abed.org.br/ congresso2017/trabalhos/pdf/213.pdf. Fecha de consulta: 30 jul. 2020. 
- Creador y criatura: percepción de profesores de español sobre la producción de material didáctico

DUARTE, R. Entrevistas em pesquisas qualitativas. Educar, Curitiba: Editora UFPR, n. 24, p. 213-225, 2004.

ERES FERNÁNDEZ, I. G. M. La producción de materiales didácticos de español lengua extranjera en Brasil. In: Anuario brasileño de estudios hispánicos: Suplemento - El hispanismo en Brasil. Embajada de España en Brasil - Consejería de Educación y Ciencia. Brasília: Thesaurus Editora de Brasília, 2000, p. 59-80. Disponível em: https://sede. educacion.gob.es/publiventa/descarga.action?f_codigo_agc=15654. Fecha de consulta: 30 jul. 2020.

FREIRE, P. Pedagogia do oprimido. Rio de Janeiro: Paz e Terra, 2019. Edición Kindle.

FREIRE, P. Pedagogia da autonomia: saberes necessários à prática educativa. São Paulo: Paz e Terra, 1996.

GATTI, B. A. Formação de professores no Brasil: características e problemas. Educ. Soc., Campinas, v. 31, n. 113, p. 1355-1379, out./dez. 2010. Disponível em: https://www.scielo. br/pdf/es/v31n113/16.pdf. Fecha de consulta: 25 ago. 2020.

GATTI, B. A; BARRETTO, E. S. S. Professores do Brasil: impasses e desafios. Brasília, DF: UNESCO, 2009.

GIL, A. C. Métodos e técnicas de pesquisa social. 8. ed. São Paulo: Atlas, 2010.

LARSEN-FREEMAN, D. Chaos/complexity science and second language acquisition. Annual Review of Apllied Linguistics, Oxford, v. 18, n. 2, p. 141-165, 1997.

LEFFA, V. J. Como produzir materiais para o ensino de línguas. In: LEFFA, V. J. (org.). Produção de materiais de ensino: teoria e prática. 2. ed. Pelotas: EDUCAT, 2007. p. 1541. Disponível em: http://www.leffa.pro.br/textos/trabalhos/prod_mat.pdf. Fecha de consulta: 10 jun. 2019.

MONTE MÓR, W. A Profissionalização do Professor de Línguas Estrangeiras e o Projeto Educacional. In: TONELLI, J. R. A.; BRUNO, F. A. T. C. (org.). Ensino-Aprendizagem de Inglês e Espanhol no Brasil: práticas, desafios e perspectivas. Coleção NPLA, v. 43, p. 2141, 2015. 
MORIN, E. O método 1: a natureza da natureza. Tradução Ilana Heineberg. Porto Alegre: Sulina, 2016.

MORIN, E. Introdução ao pensamento complexo. 4. ed. Porto Alegre: Sulina, 2015.

OLIVEIRA, R. A. de. Complexidade: conceitos, origens, afiliações e evoluções. In: PAIVA, V. L. M. de O.; NASCIMENTO, M. (org.). Sistemas Adaptativos Complexos: lingua(gem) e aprendizagem. Campinas: Pontes Editores, 2011. p. 13-34.

PAIVA, V. L. M. de O. e. Caos, complexidade e aquisição de segunda língua. In: PAIVA, V. L. M. de O.; NASCIMENTO, M (org.). Sistemas Adaptativos Complexos: Lingua(gem) e Aprendizagem. Campinas: Pontes Editores, 2011. p. 187-203.

SABOTA, B. A formação do professor de LE perante os desafios da complexidade: um convite à reflexão. In: ENCONTRO ESTADUAL DE DIDÁTICA E PRÁTICA DE ENSINO, 4. 2011, Goiânia. Anais do IV Encontro Estadual de Didática e Prática de Ensino. Goiânia: PUC-Goiás, 2011, n. p. Disponível em: http://cepedgoias.com.br/edipe/ivedipe/pdfs/ conferencia_de_abertura_de_gts/literatura_estrangeira_gtlingua.pdf. Fecha de consulta: 15 out. 2020 .

SALABERRI RAMIRO, S. El libro de texto: selección y explotación. In: BELLO, P. et al. Didáctica de segundas lenguas: estrategias y recursos. Madrid: Santillana, 1990. p. 109-123.

SILVA, L. M. P. Enseñanza de español para brasileños: elaboración de material didáctico. In: SEDYCIAS, J. (org.). O ensino de espanhol no Brasil: passado, presente, futuro. São Paulo: Parábola Editorial, 2005. p. 182-194.

SOUZA, L. M. T. M. de. Educação linguística: repensando os conceitos de língua e linguagem. In: FERRAZ, D. de M.; KAWACHI-FURLAN, C. J. (org.). Bate-papo com educadores linguísticos: letramentos, formação docente e criticidade. São Paulo: Pimenta Cultural, 2019. p. 291-306.

VETROMILLE-CASTRO, R. A entropia sociointerativa e a sala de aula de (formação de professores de) língua estrangeira: reflexões sobre um sistema complexo. In: PAIVA, V. L. M. de O.; NASCIMENTO, M. (org.). Sistemas Adaptativos Complexos: lingua(gem) e aprendizagem. Campinas: Pontes Editores, 2011. p. 113-129. 
- Creador y criatura: percepción de profesores de español sobre la producción de material didáctico

ZABALZA, M. A. O estágio e as práticas em contextos profissionais na formação universitária. Tradução Sandra Trabucco Valenzuela. São Paulo: Cortez, 2014.

COMO CITAR ESTE ARTIGO: MIRANDA, Ana Karla Pereira de; GOMES, Álvaro José dos Santos; KANASHIRO, Daniela Sayuri Kawamoto. Creador y criatura: percepción de profesores de español sobre la producción de material didáctico. Revista do GEL, v. 17, n. 3, p. 164-187, 2020. Disponível em: https://revistadogel. gel.org.br/

DOI: http://dx.doi.org/10.21165/gel.v17i3.3005

Submetido em: 16/10/2020 | Aceito em: 11/11/2020. 